Indian J. Pure Appl. Math., 44(5): 673-681, October 2013

(c) Indian National Science Academy

\title{
DIFFERENTIAL POLYNOMIAL RINGS WHICH ARE GENERALIZED ASANO PRIME RINGS
}

\author{
M. R. Helmi ${ }^{*}$ H. Marubayashi**1 and A. Ueda*** \\ * Departement of Mathematics, Andalas University, \\ Padang, West Sumatera, 25163, Indonesia \\ ** Faculty of Sciences and Engineering, Tokushima Bunri University, \\ Sanuki, Kagawa, 769-2193, Japan \\ *** Department of Mathematics, Shimane University, \\ Matsue, Shimane, 690-8504 Japan \\ e-mails:monika@fmipa.unand.ac.id; marubaya@kagawa.bunri-u.ac.jp \\ ueda@riko.shimane-u.ac.jp
}

(Received 18 July 2012; accepted 16 January 2013)

Let $R[x ; \delta]$ be a differential polynomial ring over a prime Goldie ring $R$ in an indeterminate $x$, where $\delta$ is a derivation of $R$. In this paper, we describe explicitly the group of $\delta$-stable $\mathrm{v}$ - $R$-ideals and using this results, we show that $R[x ; \delta]$ is a generalized Asano prime ring if and only if $R$ is a $\delta$-generalized Asano prime ring.

Key words : Differential polynomial ring; generalized Asano prime ring; prime Goldie ring; $\delta$-stable v-ideal.

\footnotetext{
${ }^{1}$ The second author was supported by Grant-in-Aid for Scientific Research (No. 21540056) of Japan Society for the Promotion of Science.
} 


\section{INTRODUCTION}

Throughout this paper, $R$ denotes a prime Goldie ring with simple Artinian quotient ring $Q$ (in other word, $R$ is an order in a simple Artinian ring $Q$ ) and $R[x ; \delta]$ is a differential polynomial ring over $R$ in an indeterminate $x$ with multiplication $x a=a x+\delta(a)$, where $\delta$ is a derivation of $R$.

We define the concept of $\delta$-Krull prime rings and prove that $R[x ; \delta]$ is a Krull prime ring if and only if $R$ is a $\delta$-Krull prime ring. We also determine the set of all maximal v-ideals of $R[x ; \delta]$ when it is a Krull prime ring.

In [8], we defined a notion of a $\sigma$-generalized Asano prime ring motivated by [1] and [2] and proved that a skew polynomial ring $R[x ; \sigma]$ over $R$ in an indeterminate $x$ is a generalized Asano prime ring if and only if $R$ is a $\sigma$-generalized Asano prime ring, where $\sigma$ is an automorphism of $R$.

In this paper, we define a notion of $\delta$-generalized Asano prime rings that are $\delta$-Krull prime rings whose $\delta$-v-ideals are invertible. We obtain that $R[x ; \delta]$ is a generalized Asano prime ring if and only if $R$ is a $\delta$-generalized Asano prime ring, which is proved by using the complete description of maximal v-ideals of $R[x ; \delta]$.

We refer readers to [9] or [10] for details of maximal orders and $R$-ideals.

\section{2. $\delta$-Generalized Asano Prime Rings}

First we introduce some notation. For any right (left) $R$-ideals $I(J)$, let

$$
(R: I)_{l}=\{q \in Q \mid q I \subseteq R\} \quad\left((R: J)_{r}=\{q \in Q \mid J q \subseteq R\}\right)
$$

which is a left (right) $R$-ideal and

$$
I_{v}=\left(R:(R: I)_{l}\right)_{r} \quad\left({ }_{v} J=\left(R:(R: J)_{r}\right)_{l}\right)
$$

which is a right (left) $R$-ideal containing $I(J) . I(J)$ is called a right (left) v-ideal if $I_{v}=I\left({ }_{v} J=J\right)$. In case $I$ is a two-sided $R$-ideal, it is said to be a v-ideal if $I_{v}=I={ }_{v} I$. 
Let $E(Q / R)$ be the injective hull of a right $R$-module $Q / R$ and let $F(\tau)$ be a right Gabriel topology corresponding to the torsion theory cogenerated by $E(Q / R)$. Then

$$
F(\tau)=\left\{H: \text { right ideal of } R \mid\left(R: r^{-1} H\right)_{l}=R \text { for any } r \in R\right\}
$$

where $r^{-1} H=\{a \in R \mid r a \in H\}$ by [11, Proposition 5.5, p.147]. For a right ideal $I$ of $R$, let

$$
\operatorname{cl}(I)=\{r \in R \mid r H \subseteq I \text { for some } H \in F(\tau)\} .
$$

If $\operatorname{cl}(I)=I$, then $I$ is said to be $\tau$-closed. We note that $I \subseteq \operatorname{cl}(I) \subseteq I_{v}$ for any right $R$-ideal of $R$ and so right v-ideals are $\tau$-closed. $R$ is called a right $\tau$ Noetherian ring if $R$ satisfies the a.c.c. on $\tau$-closed right ideals. Similarly we define $\tau$-closed left ideals and left $\tau$-Noetherian rings and $R$ is said to be $\tau$-Noetherian if it is right and left $\tau$-Noetherian. We note that if $R$ is right or left $\tau$-Noetherian, then $R$ satisfies the a.c.c. on right or left v-ideals of $R$.

Let $\delta$ be a derivation of $R$, that is, $\delta(a b)=\delta(a) b+a \delta(b)$ for $a, b \in R$. Then $\delta$ is extended to a derivation of $Q$ by $\delta\left(a c^{-1}\right)=\delta(a) c^{-1}-a c^{-1} \delta(c) c^{-1}$, where $a \in R$ and $c$ is a regular element of $R$.

An $R$-ideal $I$ is called a $\delta$-stable ideal (or a $\delta$-ideal for short) if $\delta(I) \subseteq I$. $R$ is called a $\delta$-Krull prime ring if it is a $\tau$-Noetherian and a $\delta$-maximal order in $Q$, that is, $O_{l}(A)=R=O_{r}(A)$ for any $\delta$-ideal $A$ of $R$, where

$$
O_{l}(A)=\{q \in Q \mid q A \subseteq A\} \text { and } O_{r}(A)=\{q \in Q \mid A q \subseteq A\}
$$

In case $\delta=0, R$ is said to be a Krull prime ring. Then we have the following.

Proposition $2.1-R$ is a $\delta$-Krull prime ring if and only if $S=R[x ; \delta]$ is a Krull prime ring.

Proof : By [9, Proposition 2.3.15], $R$ is $\tau$-Noetherian if and only if $R[x ; \delta]$ is $\tau$-Noetherian. Suppose $R$ is a $\delta$-Krull prime ring and let $A$ be a non-zero ideal of 
$S$. Then $A^{\prime}=A T$, where $T=Q[x ; \delta]$, is an ideal of $T$ by [6, Theorem 9.20 (a)]. Let $q \in O_{l}(A)$, that is $q A \subseteq A$. Then $q A^{\prime} \subseteq A^{\prime}$ and $q \in O_{l}\left(A^{\prime}\right)=T$ since $T$ is a principal ideal ring. Write $q=q_{n} x^{n}+\cdots+q_{1} x+q_{0}$, where $q_{i} \in Q$ and let

$$
L(A)=\left\{a_{l} \in R \mid \exists f(x)=a_{l} x^{l}+\cdots+a_{1} x+a_{0} \in A\right\},
$$

which is a $\delta$-ideal of $R$. For any $a \in L(A)$, there is an $a(x)=a x^{l}+\cdots+a_{1} x+a_{0} \in$ $A$ and $q a(x) \in A$ implies $q_{n} a \in L(A)$, that is $q_{n} L(A) \subseteq L(A)$. Hence $q_{n} \in R$. Continuing this method, we have $q \in S$ and similarly $O_{r}(A)=S$. Hence $S$ is a maximal order and so it is a Krull prime ring.

Conversely suppose that $S$ is a Krull prime ring and let $\mathfrak{a}$ be a $\delta$-ideal of $R$. Then $A=\mathfrak{a}[x ; \delta]$ is an ideal of $S$. Let $q \in O_{l}(\mathfrak{a})$. Then $q A \subseteq A$ and so $q \in$ $S \cap Q=R$. Similarly $O_{r}(\mathfrak{a})=R$ since $S=R[x ;-\delta]=\left\{x^{n} a_{n}+\cdots+a_{1} x+a_{0} \mid\right.$ $\left.a_{i} \in R\right\}$. Hence $R$ is a $\delta$-maximal order and so it is a $\delta$-Krull prime ring.

If $R$ is a $\delta$-Krull prime ring, then for any $\delta$-ideal $A$,

$$
(R: A)_{l}=A^{-1}=\{q \in Q \mid A q A \subseteq A\}=(R: A)_{r}
$$

and so $A_{v}=A^{-1-1}={ }_{v} A$ follows.

Let $D_{\delta}(R)$ be the set of all $\delta$-v-ideals. For $A, B \in D_{\delta}(R)$, we define $A \circ B=$ $(A B)_{v}$. The following lemma is proved in the standard way ([9, Theorem 2.1.2]). However we give a complete proof for reader's convenience.

Lemma 2.2 - If $R$ is a $\delta$-Krull prime ring, then $D_{\delta}(R)$ is a free abelian group generated by maximal $\delta$-v-ideals of $R$ with multiplication $\circ$.

Proof : Let $\mathfrak{m}$ be any maximal $\delta$-v-ideal of $R$. Then $\mathfrak{m}$ is v-invertible, that is $\mathfrak{m}^{-1} \circ \mathfrak{m}=R=\mathfrak{m} \circ \mathfrak{m}^{-1}$ because $R \supseteq \mathfrak{m}^{-1} \mathfrak{m} \supset \mathfrak{m}$ and $R \supseteq \mathfrak{m m}^{-1} \supset \mathfrak{m}$.

For any different maximal $\delta$-v-ideals $\mathfrak{m}$ and $\mathfrak{n}$ of $R$, we claim that $\mathfrak{m} \circ \mathfrak{n}=$ $\mathfrak{m} \cap \mathfrak{n}=\mathfrak{n} \circ \mathfrak{m}$. From $\mathfrak{m} \supset \mathfrak{a}=\mathfrak{m} \cap \mathfrak{n} \supseteq \mathfrak{m} \mathfrak{n}$, we have $R \supset \mathfrak{m}^{-1} \circ \mathfrak{a} \supseteq v\left(\mathfrak{m}^{-1} \mathfrak{m} \mathfrak{n}\right)=\mathfrak{n}$ and so $\mathfrak{m}^{-1} \circ \mathfrak{a}=\mathfrak{n}$. Thus $\mathfrak{a}=\mathfrak{m} \circ \mathfrak{n}$ and similarly $\mathfrak{a}=\mathfrak{n} \circ \mathfrak{m}$.

Next we will show that any $\delta$-v-ideal of $R$ is a finite product of maximal $\delta$-videals of $R$. Assume, on the contrary, that there is a $\delta$-v-ideal $\mathfrak{a}$ of $R$ which is not 
a finite product of maximal $\delta$-v-ideals of $R$. Choose a maximal with this property. Then $\mathfrak{a}$ is not a maximal $\delta$-v-ideal of $R$. Let $\mathfrak{m}$ be a maximal $\delta$-v-ideal of $R$ with $\mathfrak{m} \supset \mathfrak{a}$. Then $R \supseteq \mathfrak{m}^{-1} \circ \mathfrak{a} \supset \mathfrak{a}$ and so $\mathfrak{m}^{-1} \circ \mathfrak{a}$ is a finite product of maximal $\delta$-v-ideals of $R$. Thus so is $\mathfrak{a}$, which is a contradicion.

Finally let $\mathfrak{a}$ be any $\delta$-v-ideal and $\mathfrak{b}=\{r \in R \mid r \mathfrak{a} \subseteq R\}$. Then $\mathfrak{b}$ is a $\delta$-v-ideal of $R$ and so $\mathfrak{b}$ and $\mathfrak{b} \circ \mathfrak{a}$ are both finite product of maximal $\delta$-v-ideals of $R$. Hence $\mathfrak{a}=\mathfrak{b}^{-1} \circ(\mathfrak{b} \circ \mathfrak{a})$ is a finite product of maximal $\delta$-v-ideals $\mathfrak{m}$ of $R$ or its inverse $\mathfrak{m}^{-1}$. Thus $D_{\delta}(R)$ is a free abelian group generated by maximal $\delta$-v-ideals.

A $\delta$-ideal $P$ of $R$ is called a $\delta$-prime ideal if $I J \subseteq P$ for $\delta$-ideals $I$ and $J$ of $R$, then $I \subseteq P$ or $J \subseteq P$.

Proposition 2.3 - Suppose $S=R[x ; \delta]$ is a Krull prime ring. Then $\{\mathfrak{m}[x ; \delta]$, $M \mid \mathfrak{m}$ is a maximal $\delta-v-i d e a l$ of $R$ and $M=M^{\prime} \cap S$, where $M^{\prime}$ is a maximal ideal of $T=Q[x ; \delta]\}$ is the set of all maximal v-ideals of $S$.

Proof : Let $M$ be any maximal v-ideal of $S$ and $\mathfrak{m}=M \cap R$. It is easy to see that $M$ is a prime ideal and $\mathfrak{m}$ is a $\delta$-prime v-ideal.

(i) In case $\mathfrak{m} \neq(0)$. By [7, Lemma 1.3], $\mathfrak{m}[x ; \delta]$ is a prime ideal and it is a $\mathrm{v}$-ideal (see the proof of [12, Lemma 3]). Hence $M=\mathfrak{m}[x ; \delta]$ and $\mathfrak{m}$ is a maximal $\delta$-v-ideal since $M$ is a minimal prime ideal of $S$ by [10, Proposition 5.1.9]. Conversely suppose $\mathfrak{m}$ is a maximal $\delta$-v-ideal. Then it is a $\delta$-prime ideal and so $\mathfrak{m}[x ; \delta]$ is a prime v-ideal. Hence it is a maximal v-ideal.

(ii) In case $\mathfrak{m}=(0)$. Put $M^{\prime}=M T$, a proper ideal of $T$. To prove that $M^{\prime}$ is a maximal ideal of $T$, let $N^{\prime}$ be a maximal ideal of $T$ with $N^{\prime} \supseteq M^{\prime}$, Then $N^{\prime}=N T$, where $N=N^{\prime} \cap S$. Since $S$ is $\tau$-Noetherian and $T$ is a principal ideal ring, it follows that

$$
\begin{aligned}
N^{\prime} & =N_{v}^{\prime}=\left(T:\left(T: N^{\prime}\right)_{l}\right)_{r}=\left(T: T(S: N)_{l}\right)_{r} \\
& =\left(S:(S: N)_{l}\right)_{r} T=N_{v} T .
\end{aligned}
$$

Thus $N$ is a v-ideal and so $N=M$. Hence $M^{\prime}$ is a maximal ideal of $T$ with $M=M^{\prime} \cap S$. 
Conversely if $M^{\prime}$ is a maximal ideal of $T$, then $M=M^{\prime} \cap S$ is a maximal $\mathrm{v}$-ideal which is clear from the proof above.

Lemma 2.4 - Suppose $S=R[x ; \delta]$ is a Krull prime ring. If $A$ is a v- $S$-ideal contained in $T=Q[x ; \delta]$ with $\mathfrak{a}=A \cap Q \neq(0)$, then $A=\mathfrak{a}[x ; \delta]$.

Proof : By Lemma 2.2 and Proposition 2.3, $A=\mathfrak{b}[x ; \delta] \circ B$ where $\mathfrak{b}$ is a $\delta$-v-ideal and $B=\left(M_{1}^{e_{1}} \cdots M_{k}^{e_{k}}\right)_{v}$ such that $M_{i}^{\prime}=M_{i} T$ is a maximal ideal of $T$, $1 \leq i \leq k$. Thus $T=A T=(\mathfrak{b}[x ; \delta] B)_{v} T=B T$ and so $e_{1}=\cdots=e_{k}=0$. Hence $A=\mathfrak{b}[x ; \delta]$ with $\mathfrak{b}=A \cap Q=\mathfrak{a}$.

An $R$-ideal $A$ is said to be invertible if $A^{-1} A=R=A A^{-1}$. Then we call $R$ a $\delta$-generalized Asano prime ring if it is a $\delta$-Krull prime ring whose $\delta$-v-ideals are invertible. In case $\delta=0, R$ is said to be a generalized Asano prime ring. Let $R$ be a $\delta$-Krull prime ring. Then, by Lemma 2.2, $R$ is a $\delta$-generalized Asano prime ring if and only if any $\delta$-v-ideal of $R$ is invertible, which is equivalent to that any maximal $\delta$-v-ideal of $R$ is invertible.

Lemma 2.5 - Suppose $R$ is a $\delta$-generalized Asano prime ring. If $B=\left(M_{1}^{e_{1}} \cdots M_{k}^{e_{k}}\right)_{v}$ is a v-ideal of $S=R[x ; \delta]$, where $M_{i}^{\prime}=M_{i} T$ is a maximal ideal of $T$, then $B$ is invertible.

Proof : Since $B T$ is an ideal, $B T=w T$ for some central element $w$ in $T$ by [3, Remark 1, p.95] and so $w^{-1} B$ is a $\mathrm{v}$ - $S$-ideal such that $w^{-1} B \subseteq T$ with $\mathfrak{a}=w^{-1} B \cap Q \neq(0)$. It follows that from Lemma 2.4 that $w^{-1} B=\mathfrak{a}[x ; \delta]$. Hence $B=w \mathfrak{a}[x ; \delta]$ is invertible.

Now we obtain the main result of this paper.

Theorem 2.6 - Let $R$ be a prime Goldie ring in a simple Artinian ring $Q$. Then $R$ is a $\delta$-generalized Asano prime ring if and only if $S=R[x ; \delta]$ is a generalized Asano prime ring.

Proof : Suppose $R$ is a $\delta$-generalized Asano prime ring. Let $A$ be a v-ideal of $S$. Then $A=\mathfrak{a}[x ; \delta] \circ B=\mathfrak{a}[x ; \delta] B$, where $\mathfrak{a}$ is a $\delta$-v-ideal and $B$ is as in Lemma 2.5. Hence $A$ is invertible and so $S$ is a generalized Asano prime ring. 
Conversely suppose $S$ is a generalized Asano prime ring. Let $\mathfrak{a}$ be a $\delta$-v-ideal of $R$. Then $A=\mathfrak{a}[x ; \delta]$ is invertible with $A^{-1}=\mathfrak{a}^{-1}[x ; \delta]$ and so $\mathfrak{a}$ is invertible. Hence $R$ is a $\delta$-generalized Asano prime ring.

In [1] and [2], she studied Noetherian generalized Dedekind prime rings, which are generalized Asano prime rings in our sense. We give an example of nonNoetherian generalized Asano prime rings.

Lemma 2.7 - Let $D$ be a commutative domain with quotient field $K$ and $R=$ $D[\mathbf{x}]$ be a polynomial ring over $D$ in indeterminates $\mathbf{x}=\left\{x_{i}\right\}$ (we do not assume that $\left\{x_{i}\right\}$ is finite). Further let $\mathbf{x}^{\prime}$ be a finite subset of $\mathbf{x}$ and $I$ be a $D\left[\mathbf{x}^{\prime}\right]$-ideal. Then $\left(R: I\left[\mathbf{x}-\mathbf{x}^{\prime}\right]\right)_{l}=\left(D\left[\mathbf{x}^{\prime}\right]: I\right)_{l}\left[\mathbf{x}-\mathbf{x}^{\prime}\right]$ and in particular $\left(I\left[\mathbf{x}-\mathbf{x}^{\prime}\right]\right)_{v}=I_{v}\left[\mathbf{x}-\mathbf{x}^{\prime}\right]$.

Proof : It is clear $\left(D\left[\mathbf{x}^{\prime}\right]: I\right)_{l}\left[\mathbf{x}-\mathbf{x}^{\prime}\right] \subseteq\left(R: I\left[\mathbf{x}-\mathbf{x}^{\prime}\right]\right)_{l}$. Let $\alpha$ be an element in $\left(R: I\left[\mathbf{x}-\mathbf{x}^{\prime}\right]\right)_{l}$ and $K\left(\mathbf{x}^{\prime}\right)$ be the quotient field of $K\left[\mathbf{x}^{\prime}\right]$. Then $I K\left(\mathbf{x}^{\prime}\right)=K\left(\mathbf{x}^{\prime}\right)$ and $\alpha I \subseteq R$ imply $\alpha \in \alpha K\left(\mathbf{x}^{\prime}\right)=\alpha I K\left(\mathbf{x}^{\prime}\right) \subseteq R K\left(\mathbf{x}^{\prime}\right) \subseteq K\left(\mathbf{x}^{\prime}\right)\left[\mathbf{x}-\mathbf{x}^{\prime}\right]$. So we can write $\alpha=q_{l} \mathbf{x}_{\mathbf{l}}+\cdots+q_{i} \mathbf{x}_{\mathbf{i}}+\cdots+q_{0}$, where $q_{i} \in K\left(\mathbf{x}^{\prime}\right)$ and $\mathbf{x}_{\mathbf{i}}$ 's are subsets of $\mathbf{x}-\mathbf{x}^{\prime}$. Hence $q_{i} I \subseteq D\left[\mathbf{x}^{\prime}\right]$ for each $i$ and $q_{i} \in\left(D\left[\mathbf{x}^{\prime}\right]: I\right)_{l}$. Therefore $\alpha \in\left(D\left[\mathbf{x}^{\prime}\right]: I\right)_{l}\left[\mathbf{x}-\mathbf{x}^{\prime}\right]$ follows.

Lemma 2.8 - Let $D$ be a commutative Krull domain and $R=D[\mathbf{x}]$ be a polynomial ring over $D$ in indeterminates $\mathbf{x}$, where $\mathbf{x}$ is infinite or finite. Then

(1) $R$ is a Krull domain and it is not Noetherian if $\mathbf{x}$ is an infinite set.

(2) For any maximal v-ideal $P$ of $R$, there exists a finite subset $\mathbf{x}^{\prime}$ of $\mathbf{x}$ such that $P=P_{0}\left[\mathbf{x}-\mathbf{x}^{\prime}\right]$, where $P_{0}=P \cap D\left[\mathbf{x}^{\prime}\right]$ and $P_{0}$ is a maximal v-ideal of $D\left[\mathbf{x}^{\prime}\right]$.

Proof : (1) See for example [4, Theorem 4.2.9].

(2) Let $P$ be a maximal v-ideal of $R=D[\mathbf{x}]$. Then there exists a finite subset of $\mathbf{x}^{\prime}$ of $\mathbf{x}$ with $P_{0}=D\left[\mathbf{x}^{\prime}\right] \cap P \neq(0)$. If $A B \subseteq P_{0}$ for ideals $A$ and $B$ of $D\left[\mathbf{x}^{\prime}\right]$, then $A\left[\mathbf{x}-\mathbf{x}^{\prime}\right] B\left[\mathbf{x}-\mathbf{x}^{\prime}\right]=A B\left[\mathbf{x}-\mathbf{x}^{\prime}\right] \subseteq P_{0}\left[\mathbf{x}-\mathbf{x}^{\prime}\right] \subseteq P$ and so we have $A\left[\mathbf{x}-\mathbf{x}^{\prime}\right] \subseteq P$ or $B\left[\mathbf{x}-\mathbf{x}^{\prime}\right] \subseteq P$. Hence $A \subseteq P_{0}$ or $B \subseteq P_{0}$ holds and $P_{0}$ is a prime ideal of $D\left[\mathbf{x}^{\prime}\right]$. Now $P_{0}\left[\mathbf{x}-\mathbf{x}^{\prime}\right] \subseteq P$ and so $\left(P_{0}\right)_{v}\left[\mathbf{x}-\mathbf{x}^{\prime}\right]=\left(P_{0}\left[\mathbf{x}-\mathbf{x}^{\prime}\right]\right)_{v} \subseteq P_{v}=P$ by Lemma 2.7. Hence $\left(P_{0}\right)_{v}=P_{0}$ and $P_{0}$ is a prime v-ideal. Since $P$ is a minimal 
prime ideal, we have $P=P_{0}\left[\mathbf{x}-\mathbf{x}^{\prime}\right]$.

Example : Let $D$ be a generalized Dedekind domain. Then $R=D[\mathbf{x}]$ is also a generalized Dedekind domain. If $\mathbf{x}$ is infinite, then $R$ is not Noetherian.

PROOF : Since $R$ is a Krull domain, it is sufficient to prove that any maximal v-ideal is invertible. But this follows from the relation $P=P_{0}\left[\mathbf{x}-\mathbf{x}^{\prime}\right]$ because $D\left[\mathbf{x}^{\prime}\right]$ is a generalized Dedekind domain.

A generalized Asano prime ring is a Krull prime ring. But the converse does not necessarily hold (see [5, p.8, Example 1.10]).

\section{REFERENCES}

1. E. Akalan, On Generalized Dedekind prime rings, J. of Algebra, 320 (2008), 29072916.

2. E. Akalan, On rings whose reflexive ideals are principal, Comm. in Algebra 38 (2010), 3174-3180.

3. S. Amitsur, Derivations in simple rings, Proc. London Math. Soc. (3) 7 (1957), 87-112.

4. S. Balcerzyk and T. Józefiak, Commutative Noetherian and Krull Rings, Ellis Horwood, Warszawa (1989).

5. R. M. Fossum, The Divisor Class Group of a Krull Domaian, Springer-Verlag, Berlin (1973).

6. K. R. Goodearl and R. B. Warfield, JR., An Intoroduction to Noncommutative Noetherian Rings, London Mathematical Society, Cambridge (1989).

7. K. Kishimoto, H. Marubayashi and A. Ueda, An Ore extension over a v-HC order, J. of Okayama Unv., 27 (1985), 107-120.

8. H. Marubayashi, Intan Muchtadi-Alamsyah and A. Ueda, Skew polynomial rings which are generalized Asano prime rings, J. Algebra Appl., 12 (2013), 7.

9. H. Marubayashi and F. Van Oystaeyen, Prime Divisors and Non-commutative Valuation Rings, Lecture Notes in Math. 2059, Springer (2012). 
10. J. C. McConnell and J.C. Robson, Noncommutative Noetherian Rings, Wiley, Chichester (1987).

11. B. Stenström, Rings of Quotients, Grundlehren Math. 217, Springer-Verlag, Berlin, (1975).

12. Y. Wang, H. Marubayashi, E. Suwastika and A. Ueda, The group of divisors of an Ore extension over a Noetherian integrally closed domain, The Aligarh Bulletin of Math., 29 (2) (2010), 149-152. 